COLLOIDS AND SURFACES B-BIOINTERFACES (ISSN: 0927-7765) 118: (1) pp. 164171. (2014)

DOI: 10.1016/j.colsurfb.2014.03.048

\title{
A mechanistic view of lipid membrane disrupting effect of PAMAM dendrimers
}

\author{
Szilvia Berényi ${ }^{a{ }^{*}}$, Judith Mihály ${ }^{a 2}$, András Wacha ${ }^{a 2}$, Orsolya Töke ${ }^{b}$, Attila Bóta ${ }^{a 2}$ \\ a Dept. of Biological Nanochemistry, Institute of Molecular Pharmacology, Research Centre of Natural Sciences, \\ Hungarian Academy of Sciences, Magyar Tudósok körútja 2. 1117 Budapest, Hungary \\ ${ }^{b}$ Dept. of Structural Chemistry, Institute of Organic Chemistry, Research Centre of Natural Sciences, Hungarian \\ Academy of Sciences, Magyar Tudósok körútja 2. 1117 Budapest, Hungary \\ * berenyi.szilvia@mail.bme.hu \\ ${ }^{1}$ MTA-BME “Lendület” Chemical Nanosensors Research Group, Department of Inorganic and Analytical \\ Chemistry, Budapest University of Technology and Economics, Szent Gellért tér 4, 111 Budapest, Hungary \\ ${ }^{2}$ Dept. of Biological Nanochemistry, Institute of Materials and Environmental Chemistry, Research Centre of \\ Natural Sciences, Hungarian Academy of Sciences, Magyar Tudósok körútja 2. 1117 Budapest, Hungary \\ * berenyi.szilvia@mail.bme.hu
}

\section{Abstract}

The effect of $5^{\text {th }}$ generation polyamidoamine (PAMAM G5) dendrimers on multilamellar dipalmitoylphosphocholine (DPPC) vesicles was investigated. PAMAM was added in two different concentration to the lipids $\left(10^{-3}\right.$ and $10^{-2}$ dendrimer/lipid molar ratios). The thermal behavior of the evolved systems was characterized by DSC; while the structure and the morphology were investigated with small- and wide-angel X-ray scattering (SWAXS), freeze-fracture electron microscopy (FFTEM) and phosphorus-31 nuclear magnetic resonance ( $\left.{ }^{31} \mathrm{P}-\mathrm{NMR}\right)$ spectroscopy, respectively. IR spectroscopy was used to study the molecular interactions between PAMAM and DPPC. The obtained results show that the dendrimers added in $10^{-}$ ${ }^{3}$ molar ratio to the lipids generate minor perturbations in the multilamellar structure and thermal character of liposomes, while added in $10^{-2}$ molar ratio dendrimers cause major disturbance in the vesicular system. The terminal amino groups of the dendrimers are in strong interaction with the phosphate headgroups and through this binding dendrimers disrupt the regular multilamellar structure of DPPC. Besides highly swollen, fragmented bilayers, small vesicles are formed.

Keywords: dendrimer; DPPC; model membrane; infrared spectroscopy; SAXS.

\section{Introduction}

Dendrimers are highly branched, globular macromolecules with numerous terminal groups and internal cavities. The branches are repeated in a radial concentric way and each concentric layer is called a generation $(\mathrm{G})$. The higher the number of generations is the more terminal groups and bigger size the dendrimer has. The pharmacological and biomedical applicability of dendrimers has been extensively studied and reviewed lately, yet it is still a progressive field of research [1-7]. Dendrimers are ideal nanocarriers for drugs, because not only the drug itself, but also targeting and imaging molecules can be attached to the same macromolecule. Toxicity, however can limit their use, especially in the case of higher generation, positively charged dendrimers [8-11]. 


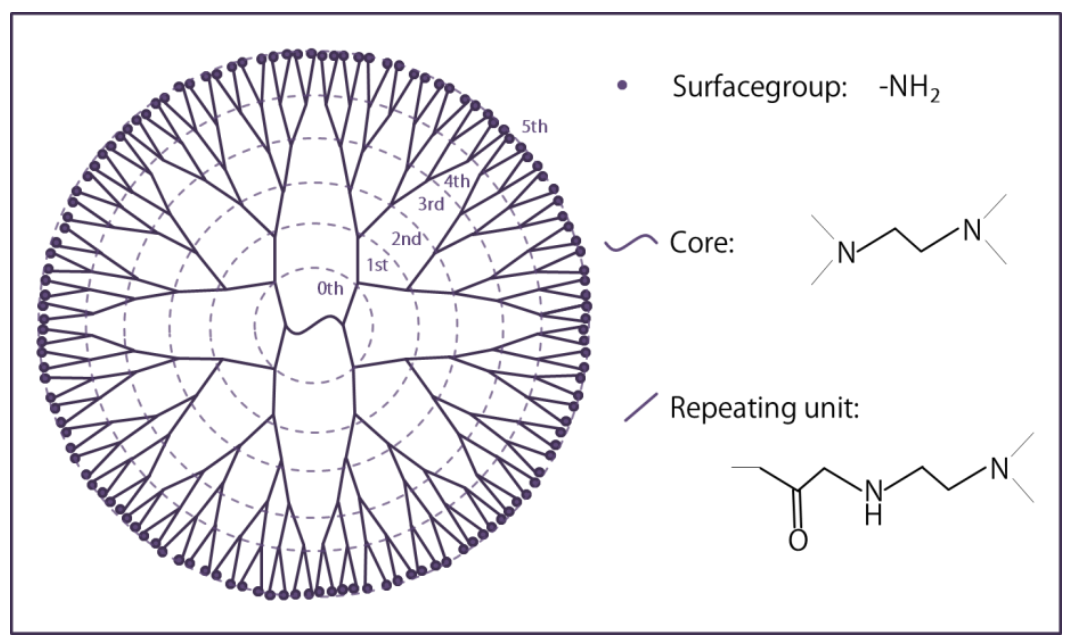

Figure 1. Schematic structure of $5^{\text {th }}$ generation polyamidoamine dendrimer with ethylenediamine core.

The interaction between cell membranes and nanocarriers, such as dendrimers, is very important, because in the most cases carriers have to get across the lipid bilayer without disrupting it. Previous studies based on atomic force microscopy (AFM) [12-17] isothermal titration calorimetry (ITC), fluorescence correlated spectroscopy (FCS) [17-18], in vitro experiments [13,19-20] and molecular dynamics simulations (MD) [14,17,21-24] showed that cationic dendrimers disrupt lipid bilayers by forming holes on the bilayer surface and may remove lipids from it. The degree of the disruption depends on the size and charge of the dendrimer. Acetylated and small generation (G3) dendrimers attach to the lipid surface without destabilizing it. Hole formation caused by positively charged dendrimers up to G5 have been found to be reversible. This is an important question regarding drug delivery and cytotoxicity, because dendrimers should penetrate into cells without causing cell injury. Most of the above mentioned studies were based on supported lipid bilayers.

Liposomes are widely used as model systems of biological membranes, since both contain lipid bilayers as their basic structural unit [12]. This way, investigating the effect of foreign molecules on the structure and thermal behavior of liposomes, we can obtain basic information on the physicochemical level of toxicity. Karoonuthaisiri et al. investigated the effect of different generation PAMAM dendrimers on the permeability of unilamellar liposomes composed of different lipids [25]. The results of their experiments indicate that PAMAM dendrimers effectively disrupt the lipid bilayer, when membranes contain also non-bilayer forming lipids (eg. dioleylphosphoethanolamine/DOPE), while they found minor effect on membranes composed only from phosphocholine lipids. Åkesson and coworkers also used unilamellar liposomes as model system and investigated the effect of G6 PAMAM dendrimers $(\mathrm{d} \approx 6.7 \mathrm{~nm})$ with dynamic light scattering, cryo-TEM and small-angle X-ray scattering (SAXS) [26]. They showed that dendrimers attach to the surface of the vesicles and can bridge neighboring vesicles until liposomes collapse in lamellar phase in which dendrimers are located between the layers. Later they investigated the effect of dendrimers on giant unilamellar vesicles (GUVs) by fluorescent microscopy and on supported lipid bilayers by quartz crystal microbalance with dissipation monitoring (QCM-D) and neutron reflectivity [27-29]. Interestingly they have found that the dendrimers enhance the permeability of the membranes for small molecules without hole formation or passive translocation across the membrane. Leaning on the results a model was interpreted in which the dendrimers are intercalated in the bilayer headgroup region and a large-scale roughness is generated as the bilayer changes curvature to follow the shape of the dendrimers.

Differential scanning calorimetry (DSC), small- and wide-angle X-ray scattering (SWAXS), infrared spectroscopy (IR) and freeze-fracture electron microscopy (FFTEM) are prevalent methods to characterize liposomes and to monitor the changes in their structure caused by other molecules [30-33]. Still there are only a few papers in which these methods are used in order to investigate the interactions between dendrimers and liposomes [34-36]. Klajnert et al. used DSC to investigate the effect of hydrophilic and hydrophobic dendrimers on liposomes. It was established that hydrophilic dendrimers would be located near to lipid headgroups, while 
hydrophobic dendrimers interact with the alkyl chains of the lipids and may cause loss of integrity in the membrane [34]. Gardikis et al. examined the incorporation of G4 and G3.5 polyamidoamine (PAMAM) dendrimers $\left(\mathrm{d}_{\mathrm{G} 4} \approx 4.5 \mathrm{~nm}, \mathrm{~d}_{\mathrm{G} 3.5} \approx 4 \mathrm{~nm}\right)$ in dipalmitoylphosphatidylcholine (DPPC) bilayers using DSC and Raman spectroscopy. They found that the maximum percentage of PAMAM dendrimers that can incorporate into multilamellar liposomes is $5 \mathrm{~mol} \%$ for G4 and $3.5 \mathrm{~mol} \%$ for G3.5 [35]. Wrobel et al. investigated the effect of positively charged phosphorous-containing dendrimers on unilamellar liposomes by measuring fluorescence anisotropy and DSC and showed that dendrimers interact with both hydrophobic and hydrophilic parts of the bilayer [36]. Smith and coworkers investigated the effect of G5 and G7 PAMAM dendrimers on multilamellar vesicles by solid-state NMR techniques. They have found that dendrimers have higher impact on alkyl chain region than on headgroups; besides they used partially hydrated or highly concentrated, fully hydrated membranes [37].

In this study the interaction between positively charged fifth generation (G5) PAMAM dendrimers and DPPC based liposomes were investigated in order to reveal what happens between dendrimers and lipids when the size of the dendrimer is not big enough to form a liposome-coated dendrimer complex. Mecke and coworkers have made thermodynamic calculations and proposed that G7 PAMAM dendrimers $(\mathrm{d} \approx 8 \mathrm{~nm})$ are big enough to have a closed lipid bilayer wrapping them [14]. Although the diameter of $\mathrm{G} 5(\mathrm{~d} \approx 5.5 \mathrm{~nm})$ dendrimers is too small for this, they still remove lipids from PC membranes. We aimed to understand this interaction in depth.

For a comprehensive characterization we have combined DSC, SWAXS, FFTEM, phosphorus-31 nuclear magnetic resonance $\left({ }^{31} \mathrm{P}-\mathrm{NMR}\right)$ and infrared spectroscopy. Thermal characterization with DSC provides information on the lipid membrane phase transition temperatures and changes in enthalpy. Structural information can be obtained using X-ray scattering techniques as one can deduce the lamellarity from a SAXS pattern of a liposomal system and establish the characteristic distances between lamellae, while WAXS patterns provide information about subcells in the alkyl chain region $[31,38]$. FFTEM enables direct visualization of lipid structures when examining a replica made on the freeze-fractured surface of the sample. ${ }^{31} \mathrm{P}$-NMR spectroscopy can give information of the phase behavior of the lipids as the shape of the signal is characteristic for the different isotropic and anisotropic phases. [39, 40] Infrared spectroscopy is used to study the structure and organization of lipid bilayers. Changes in the frequencies and widths of vibrational bands and/or splitting of the spectral features provide information on the interactions between functional groups of lipids and macromolecules $[32,33]$.

\section{Materials and methods}

PAMAM G5 (PAMAM) dendrimer (with ethylenediamine core, MW: $28824 \mathrm{~g} / \mathrm{mol}, \mathrm{d} \approx 5.5 \mathrm{~nm}$, Fig. 1 ) in $5 \mathrm{wt} \%$ methanol solution was purchased from Sigma-Aldrich. Highly purified synthetic 1,2-dipalmitoyl-sn-glycero-3-phosphocholine (DPPC) was purchased from NOF Corporation. All materials were used without further purification.

Mixtures of PAMAM dendrimers and DPPC were prepared at $10^{-3}$ and $10^{-2}$ dendrimer/lipid molar ratio. The methanol solution of the dendrimer was added to the DPPC, and then pure chloroform was added until all of the lipids were dissolved. The mixture was dried under vacuum. Millipore water was added to the dried films, so that the lipid/water ratio was $20 \mathrm{wt} \%$. Hydration was followed by heating up to $60{ }^{\circ} \mathrm{C}$, cooling down to $4{ }^{\circ} \mathrm{C}$, reheating up to $60{ }^{\circ} \mathrm{C}$ and vortexing intensively. This procedure was repeated several times to achieve homogeneous dispersions.

\subsection{Differential scanning calorimetry}

Samples were examined with a Setaram $\mu \mathrm{DSC} 3$ evo apparatus. All samples were scanned three times from $25^{\circ} \mathrm{C}$ to $70{ }^{\circ} \mathrm{C}$. Scanning rate was $1{ }^{\circ} \mathrm{C} / \mathrm{min}$ first time, then $0.5{ }^{\circ} \mathrm{C} /$ min during the heating and $1{ }^{\circ} \mathrm{C} / \mathrm{min}$ during cooling period. All measurements were repeated after $30 \mathrm{~min}$ incubation at $25^{\circ} \mathrm{C}$. As a reference an empty sample holder was used. The sample quantity used for DSC measurements was about $10 \mathrm{mg}$ in each case. 


\subsection{Small-and wide-angle X-ray scattering}

Small- and wide-angle X-ray scattering measurements were performed using a modified compact Kratky-type camera with slit collimation, thus the intensity curves of SAXS were corrected considering the geometry of the beam profile. With this SWAXS camera simultaneous detection can be performed in the small- and wide-angle regions. A Cu-anode sealed X-ray tube provided the X-ray source. The scattering of Ni-filtered CuK $\alpha$ radiation ( $\lambda=1.542 \AA)$ was recorded in the small-angle range. The scattering variable is defined as $q=4 \pi \sin \Theta / \lambda$, where $2 \Theta$ is the scattering angle. In the case of periodic samples the position of the first Bragg-peak is $q=2 \pi / d$, where $d$ is the characteristic periodicity of the sample. Samples were filled into thin-walled quartz capillaries with $1 \mathrm{~mm}$ average diameter. For scattering experiments each capillary was transferred into a metal capillary holder, which was placed into an aluminum block. This block was built directly into the beamline and was used as a thermal incubator for controlled annealing at different temperatures. After $15 \mathrm{~min}$ incubation of the samples, $1000 \mathrm{~s}$ expositions were made by using two MBraun PSD-50 type linear position sensitive detectors (MBraun, Garching, Germany) in the small- and wide-angel ranges, respectively.

Some measurements were performed at the synchrotron beamline B1 of the storage-ring DORIS III in HASYLAB/DESY, Hamburg, Germany [41-43]. SAXS patterns were recorded with a Pilatus 300k positionsensitive detector (Dectris Ltd, Switzerland) [44]. Later, SAXS was re-measured with a Pilatus 1M detector [4546]. The beam used was point-collimated $(1 \times 0.7 \mathrm{~mm})$ and monochromatized to $9.6 \mathrm{keV}$ or $12 \mathrm{keV}$ (repeated SAXS measurement) with a Si (311) double crystal monochromator. Radial scattering curves were calculated from the images and normalized into absolute units according to the pre-calibrated glassy carbon reference available at the beamline.

\subsection{Freeze-fracture transmission electron microscopy}

Freeze-fracture electron microscopy was used for direct visualization of the evolved structures. The gold sample holders used for freeze-fracturing were incubated at $25{ }^{\circ} \mathrm{C}$ or at $50{ }^{\circ} \mathrm{C}$, at the same temperatures as the samples. Droplets of 1-2 $\mu \mathrm{l}$ of the sample were pipetted onto the gold sample holder and frozen by plunging it immediately into partially solidified Freon for 20 seconds and stored in liquid nitrogen. Fracturing was performed at $-100{ }^{\circ} \mathrm{C}$ in a Balzers freeze-fracture device (Balzers BAF $400 \mathrm{D}$, Balzers AG, Vaduz, Liechtenstein). Replicas of the fractured surfaces etched at $-100{ }^{\circ} \mathrm{C}$ were made by platinum-carbon shadowing, then cleaned with a water- surfactant solution and washed with distilled water. The replicas were placed on 200 mesh copper grids and examined in a MORGANI 268D transmission electron microscope.

\subsection{NMR measurements}

NMR experiments were carried out at 25 and $50{ }^{\circ} \mathrm{C}$ on $400 \mathrm{MHz}$ (for $1 \mathrm{H}$ ) Varian NMR SYSTEM spectrometer using a $5 \mathrm{~mm}$ direct detection dual-broadband ${ }^{15} \mathrm{~N}-{ }^{31} \mathrm{P} /\left\{{ }^{1} \mathrm{H}-{ }^{19} \mathrm{~F}\right\}$ probe equipped with a $\mathrm{Z}$ pulse field gradient. Phosphorous spectra were recorded with $2 \mathrm{~s}$ recycle delay and WALTZ proton decoupling. ${ }^{31} \mathrm{P}$ shifts are given relative to the external reference of $85 \% \mathrm{H}_{3} \mathrm{PO}_{4}$.

\subsection{Infrared spectroscopy}

IR measurements were performed on a Varian FTS-2000 (Scimitar Series) FTIR spectrometer (Varian Inc., US) equipped with a 'Golden Gate' single reflection diamond ATR accessory (Specac, UK). The ATR cell was modified with a custom-made liquid cell cover permitting the whole sample holder to be thermostated by circulating water. About $5 \mu \mathrm{l}$ liposome suspension was spread on the surface of the diamond ATR element and the temperature was equilibrated for $5 \mathrm{~min}$ before spectrum collection. All spectra were recorded at a resolution of $2 \mathrm{~cm}^{-1}$ by co-addition of 256 individual scans. ATR correction and water background subtraction was applied for all spectra. Spectral manipulations, including also curve fitting procedure, were performed by means of the GRAMS/32 software package. For curve fitting, band positions were estimated using the second derivative, and band shapes were approximated by Lorentzian functions until the minimum of $\chi^{2}$ was reached. 


\section{Results and discussions}

\subsection{Changes in thermal behavior}

DPPC forms multilamellar vesicles (MLVs) in water spontaneously. The MLVs may vary in size between a few tens of nanometers and a few micrometers. The fully hydrated DPPC/water system exhibits three mesomorphic phases in the examined temperature interval: gel state $\left(\mathrm{L}_{\beta^{\prime}}\right)$, rippled gel state $\left(\mathrm{P}_{\beta^{\prime}}\right)$ and liquid crystalline state $\left(\mathrm{L}_{\alpha}\right)$. Phase transition between $\mathrm{L}_{\beta^{\prime}}$ and $\mathrm{P}_{\beta^{\prime}}$ is called pretransition and occurs around $35{ }^{\circ} \mathrm{C}$ mainly due to the rotation of the polar headgroups. The pretransition has a relatively small change in enthalpy. The main transition from gel to liquid crystalline phase is due to the cooperative melting of the hydrocarbon chains associated with changes of $\mathrm{C}-\mathrm{C}$ single bonds conformations from trans to gauche. The main transition has a well-defined transition temperature $\left(41.1^{\circ} \mathrm{C}\right)$ and change in enthalpy $(36.5 \mathrm{~kJ} / \mathrm{mol})$ [31]. The shape of the main transition peak refers to the cooperativity of the chain melting, i.e., a sharper peak indicates that more molecules are melting simultaneously [30, 47-48].

The alterations in the phase transition characteristics induced by the PAMAM dendrimers were followed by DSC. Thermograms of fully hydrated DPPC and DPPC/dendrimer systems are shown in Figure 2, and the corresponding calorimetrical data are collected in Table 1. where Tm is the transition temperature, measured by the peak maximum, $\Delta \mathrm{H}$ is the calorimetric change in enthalpy and $\Delta \mathrm{T}_{1 / 2}$ is the half width of the main transition peak. Between the thermograms recorded during two consecutive scans there were no major changes neither during the heating nor during the cooling period. In the presence of the dendrimers the shape of the endotherms alters depending on the PAMAM/lipid ratio.

PAMAM dendrimers affect the pretransition significantly when added to the lipids in $10^{-3}$ molar ratio, the temperature as well as the change in enthalpy decreases, revealing an interaction between the lipid headgroups and the dendrimers. The temperature of the main transition peak did not change, only the increased $\Delta \mathrm{T}_{1 / 2}$ compared to the pure DPPC/water system shows the effect of the dendrimers. The observation of dendrimers causing major disturbance in pretransition without changing the main transition temperature indicate that dendrimers interact with the headgroups of the phospholipids and in this way affect the chain packing slightly causing a loss in cooperativity. However, in this concentration the interaction does not perturb the lipid system substantially. Klajnert and Epand have found similar results when investigating the effect of G3 PAMAM dendrimers on DPPC MLVs [34]. They explained it as a consequence of the dendrimer inserting into the bilayer and eliminating steric crowding of the headgroups.

Increasing the amount of the dendrimers changes the thermal behavior of the lipids significantly (Fig.2.). Pretransition is completely absent in the case of $10^{-2}$ dendrimer/lipid molar ratio. Instead of a single, sharp peak there is an envelope of at least three peaks around the temperature range of the main transition of the pure lipid system. Gardikis and coworkers found similar results when they added G4 PAMAM dendrimers to DPPC in $10 \mathrm{~mol} \%$, which is higher than the concentration applied by us, but also the size and the number of terminal amine-groups of the $4^{\text {th }}$ generation dendrimer $(\mathrm{d} \approx 4.5 \mathrm{~nm})$ are smaller than of $5^{\text {th }}$ generation dendrimer. [35] The broad peak at $40.4{ }^{\circ} \mathrm{C}$ shows increased membrane fluidity and significantly decreased cooperativity of alkyl chain melting. Meanwhile the high temperature shoulder and the peak at $45.5{ }^{\circ} \mathrm{C}$ correspond presumably to lipid domains that are less hydrated [50]. It seems plausible that the system became heterogeneous, because of a strong interaction between the dendrimers and the lipids, which affects the headgroups as well as the chain region. Yet further investigations are required to trace the detailed character of this interaction. 


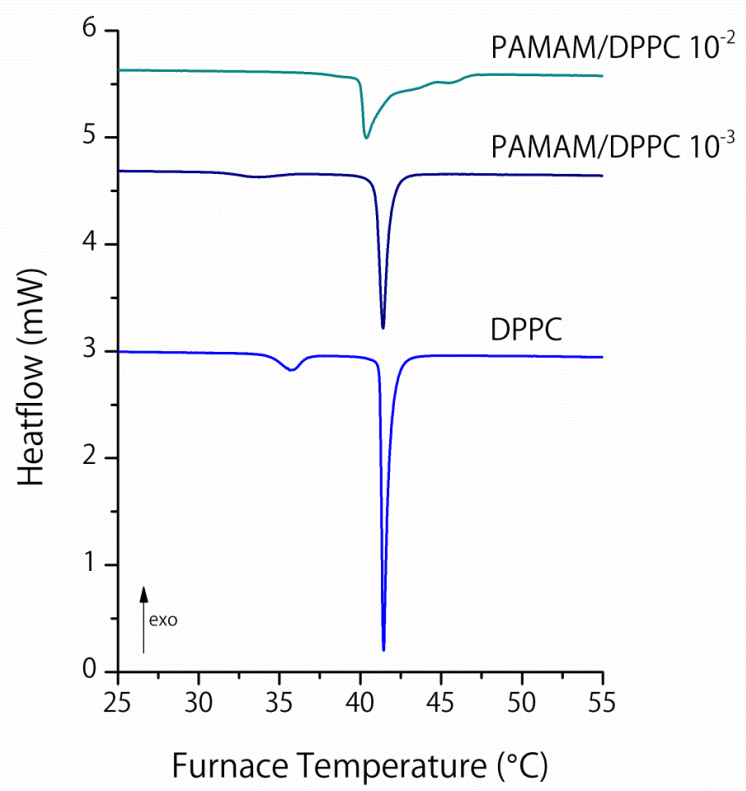

Figure 2. DSC endotherms of fully hydrated DPPC MLVs and PAMAM-loaded DPPC-systems

\begin{tabular}{lcccccc}
\hline & \multicolumn{2}{c}{ Pretransition } & \multicolumn{3}{c}{ Main transition } \\
& $\mathrm{T}_{\mathrm{m}}\left({ }^{\circ} \mathrm{C}\right)$ & $\Delta \mathrm{H}(\mathrm{kJ} / \mathrm{mol})$ & $\mathrm{T}_{\mathrm{m}}\left({ }^{\circ} \mathrm{C}\right)$ & $\Delta \mathrm{T}_{1 / 2}\left({ }^{\circ} \mathrm{C}\right)$ & $\Delta \mathrm{H}(\mathrm{kJ} / \mathrm{mol})$ \\
\hline DPPC & 35.7 & 5.4 & 41.4 & 0.4 & 36.6 \\
\hline PAMAM /DPPC & $10^{-3}$ & 33.6 & 3.4 & 41.4 & 0.6 & 36.6 \\
\hline PAMAM /DPPC & $10^{-2}$ & - & - & - & - & 45.9 \\
\hline
\end{tabular}

Table 1 Thermometric data obtained from DSC measurements of fully hydrated DPPC MLVs and PAMAMloaded DPPC-systems

\subsection{Changes in lipid membrane structure and morphology}

The structural studies were carried out at $25^{\circ} \mathrm{C}, 38^{\circ} \mathrm{C}$ and $46^{\circ} \mathrm{C}$, corresponding to the characteristic temperature range of the gel $\left(\mathrm{L}_{\beta^{\prime}}\right)$, rippled gel $\left(\mathrm{P}_{\beta^{\prime}}\right)$ and liquid crystalline phases $\left(\mathrm{L}_{\alpha}\right)$ of the fully hydrated multilamellar DPPC vesicles. SWAXS patterns (the full measured SAXS and the relevant part of the WAXS curves) of the pure lipid and the loaded systems are shown in Fig. 3. Measurements at different temperatures are grouped together for each PAMAM/DPPC ratio. In the case of $10^{-2}$ dendrimer/lipid molar ratio, only two curves are plotted, because in the absence of pretransition, scattering curves at $25{ }^{\circ} \mathrm{C}$ and $38{ }^{\circ} \mathrm{C}$ were the same. Characteristic periodicities of layer arrangements are: $6.3 \mathrm{~nm}$ in $\mathrm{L}_{\beta^{\prime}}, 7.1 \mathrm{~nm}$ in $\mathrm{P}_{\beta^{\prime}}$ and $6.5 \mathrm{~nm}$ in $\mathrm{L}_{\alpha}$ phase. These periodic distances of the layers appear as Bragg reflections in several orders in the SAXS patterns located at the values of $\mathrm{q}_{\mathrm{n}}=\mathrm{n}(2 \pi / \mathrm{d})$, where $\mathrm{n}$ is an integer, and $\mathrm{d}$ is the periodicity. Meanwhile, the repeating distances of the subcells in the alkyl chain region can be measured by WAXS. In $\mathrm{L}_{\beta^{\prime}}$ phase there is a hybrid subcell with $0.418 \mathrm{~nm}$ and $0.408 \mathrm{~nm}$ periodicities, while the characteristic size of the hexagonal subcell in $P_{\beta^{\prime}}$ is $0.414 \mathrm{~nm}$. No subcell exists in liquid crystalline phase due to the laterally disordered chain packing. SWAXS patterns (the full measured SAXS and the convenient part of the WAXS curves) of the pure lipid and the loaded systems are shown in Figure 3. These results are in agreement with the literature data [51]. 


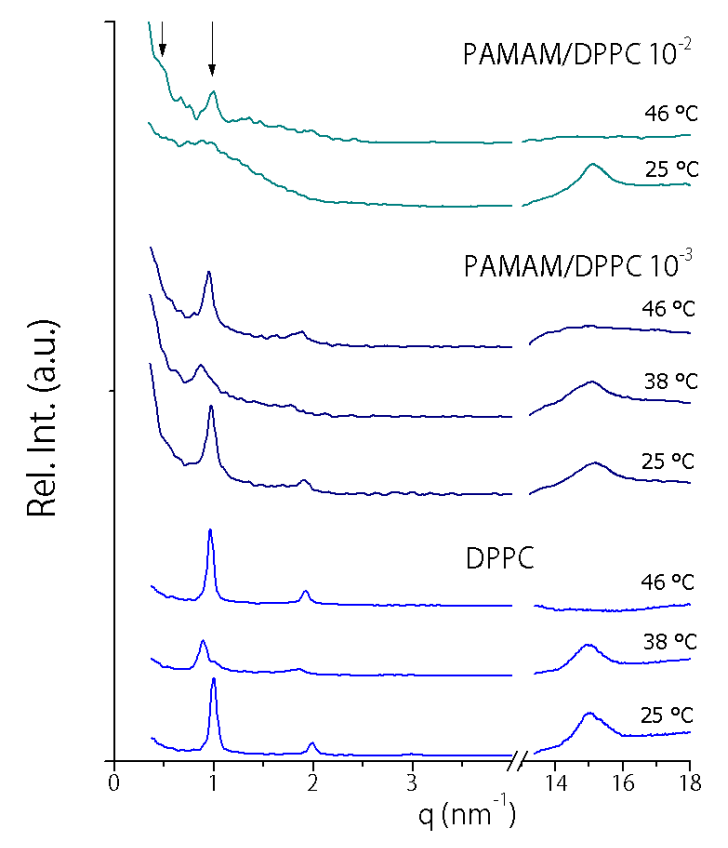

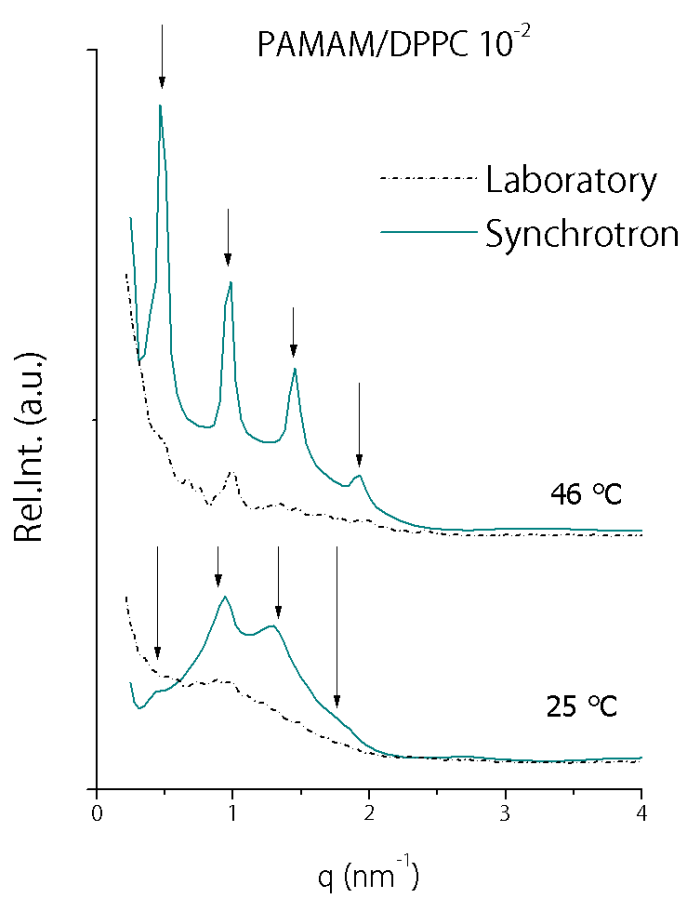

Figure 3. Left: small and wide-angle X-ray scattering curves of fully hydrated DPPC MLVs and PAMAMloaded DPPC-systems measured at different temperatures. Right: small-angle X-ray scattering curves of PAMAM-loaded DPPC-system having $10^{-2}$ dendrimer/lipid molar ratio measured at the synchrotron station and on a laboratory instrument.

The doped system with $10^{-3}$ dendrimer/lipid molar ratio exhibits the main structural features of the pure lipid system: the positions of Bragg-reflections are nearly the same as those of the pure lipid/water system $\left(\mathrm{d}_{25^{\circ} \mathrm{C}}=6.4\right.$ $\mathrm{nm}, \mathrm{d}_{38^{\circ} \mathrm{C}}=7.2 \mathrm{~nm}, \mathrm{~d}_{46}{ }^{\circ} \mathrm{C}=6.6 \mathrm{~nm}$ ), except that they are broadened. The shape of a reflection peak in a SAXS curve provides structural information: Sharper peaks refer to numerous well-ordered bilayers, while broader ones represent a loss in correlation and/or in the number of bilayers. [52] The broadening of the characteristic Bragg reflections in the case of the loaded system show the breakdown of the multilamellar correlation due to the presence of dendrimers. These findings explain the loss in cooperativity observed during the phase transitions by means of DSC method. In the gel and rippled gel phases the increase in the width of the WAXS peaks indicates a slight change in the chain packing. Instead of the complex Bragg profile corresponding to the hybrid hexagonal subcell, a wide one, centered at $\mathrm{q}_{\mathrm{n}}=2 \pi \mathrm{n} / 0.414 \mathrm{~nm}^{-1}$ can be observed at $25{ }^{\circ} \mathrm{C}$. At $38^{\circ} \mathrm{C}$ the shape of the profile remains nearly the same, meaning a decreasing difference in the chain packing between the two gel phases. The similar structure can give an explanation to the decreased phase transition enthalpy measured by the DSC.

The SAXS-patterns related to the samples with $10^{-2}$ dendrimer/lipid molar ratio differ significantly from the others. At $25{ }^{\circ} \mathrm{C}$ all Bragg reflections disappear, only a single "hump" can be observed. This part of the scattering curve may correspond to the form factor (the Fourier transform of the electron density profile) of single lipid bilayers, indicating the lack of a well-ordered multilayered structure [52-55]. However it was not possible to obtain more details on a line focus Kratky SAXS camera. Surprisingly, in the temperature domain of the liquid crystalline phase $\left(46^{\circ} \mathrm{C}\right)$ the pattern provides definite diffraction peaks, moreover, a shoulder in the beginning part of the scattering curve by particularly small $\mathrm{q}$ value $\left(2 \pi / 13 \mathrm{~nm}^{-1}\right)$ appears. Meanwhile, the character of the WAXS-pattern at $25^{\circ} \mathrm{C}$ is more similar to the pattern of the pure DPPC MLVs in contrast to what was observed in the case of PAMAM/DPPC $10^{-3}$ system. 
In order to obtain more information about the structural formations of the sample having $10^{-2}$ PAMAM/DPPC molar ratio, it was examined by SAXS method also at a synchrotron station (Fig. 3.). The advantage of the point focus geometry is the absence of artifacts arising from the desmearing procedure and that the characteristic Bragg reflections are more expressed. The results obtained at the synchrotron station are in agreement with the laboratory SAXS measurements but they also allow finer details to be resolved. Especially, the "hump" that was observed with the laboratory apparatus at $25^{\circ} \mathrm{C}$, seems to be a complex peak consisting of the form factor of the bilayers and of equidistant reflections, which may correspond to a highly swollen lamellar phase ( $\mathrm{d}=14 \mathrm{~nm})$. The higher intensity of the second order diffraction is due to its closeness in position to the maximum of the absolute square of the bilayer form factor. Presumably, this increased period is the consequence of the dendrimers embedded into the water shells between the lipid bilayers and of the electrostatic repulsion caused by the net positive charge of the dendrimers, which may lead to an extended interbilayer water layer. The fact that the form factor is clearly expressed shows that successive layers are loosely correlated and/or laterally not extended. The scattering curve does not preclude the local presence of other structures.

The SAXS curve obtained at $46^{\circ} \mathrm{C}$ exhibits equidistant Bragg reflections in four orders with a still unusually high periodic distance $(13 \mathrm{~nm})$, strengthening the results of the laboratory SAXS studies. Kelly et al. have investigated the interactions between G3 PAMAM dendrimers and DMPC bilayers in gel and liquid phase by MD simulations [23]. As they describe, dendrimers have nearly spherical shape when interacting with gel phase lipids, but when interacting with fluid phase lipids they are flattened on the bilayer surface, moreover they enter in the alkyl chain region. This change in the dendrimer morphology can explain the decreased periodic distance in the temperature domain of the liquid crystalline phase compared to that of gel phase.

To visualize the lipid structures in the presence of PAMAM dendrimers $\left(10^{-2}\right.$ dendrimer/lipid molar ratio $)$ FFTEM investigations were also performed (Fig. 4.). At $25{ }^{\circ} \mathrm{C}$ no liposome appears, but besides amorphous layers, there are domains of closely packed grains, which may be responsible for the complex scattering curve above. It is clearly shown that dendrimers hinder the formation of regular multilamellar vesicles, however we were able to detect stacks of the swollen layers, in which the periodic distance is in the range of 14-15 nm, in good agreement with the SAXS results. At $50{ }^{\circ} \mathrm{C}$ rather ragged layers can be observed, and simultaneously very small, spherical entities are present with an average diameter of 20-50 nm. These spheres are more regular forms compared to the grains of the non-lamellar domains observed at $25^{\circ} \mathrm{C}$. It seems that besides the weakly correlated, fragmented lamellae, small proportion of micelles or other highly curved vesicles are present. Since G5 PAMAM dendrimers do not have a radius high enough to support individually a surrounding vesicle [17], aggregated dendrimers should be in the core of these assemblies. The microscale inhomogeneities observed on the TEM pictures give an explanation for the complex main transition peak on the DSC curve (Fig.2.). The domains containing more dendrimers should have more significant change in the molecular packing of surrounding lipids. Åkesson et al got similar results when investigating giant liposomes by fluorescent microscopy [27].

Phospholipids in different phases provide different ${ }^{31} \mathrm{P}-\mathrm{NMR}$ spectra. $[39,40]$ In the lamellar configuration the spectrum is asymmetrical with a low-frequency peak and high-frequency shoulder; in the hexagonal phase the phospholipid spectrum has a reversed asymmetry (high-frequency peak with low-frequency shoulder); in the case of cubic or other isomorph phase the peak is narrow and symmetric. We performed ${ }^{31} \mathrm{P}-\mathrm{NMR}$ studies (Fig. 5.) and found that besides the broad, asymmetric peak corresponding to the lamellar phase of DPPC, a narrow peak appears around $0 \mathrm{ppm}$, which is still present on the spectrum measured at $50{ }^{\circ} \mathrm{C}$ and corresponds to the small proportion of highly curved vesicles seen on FFTEM pictures. 

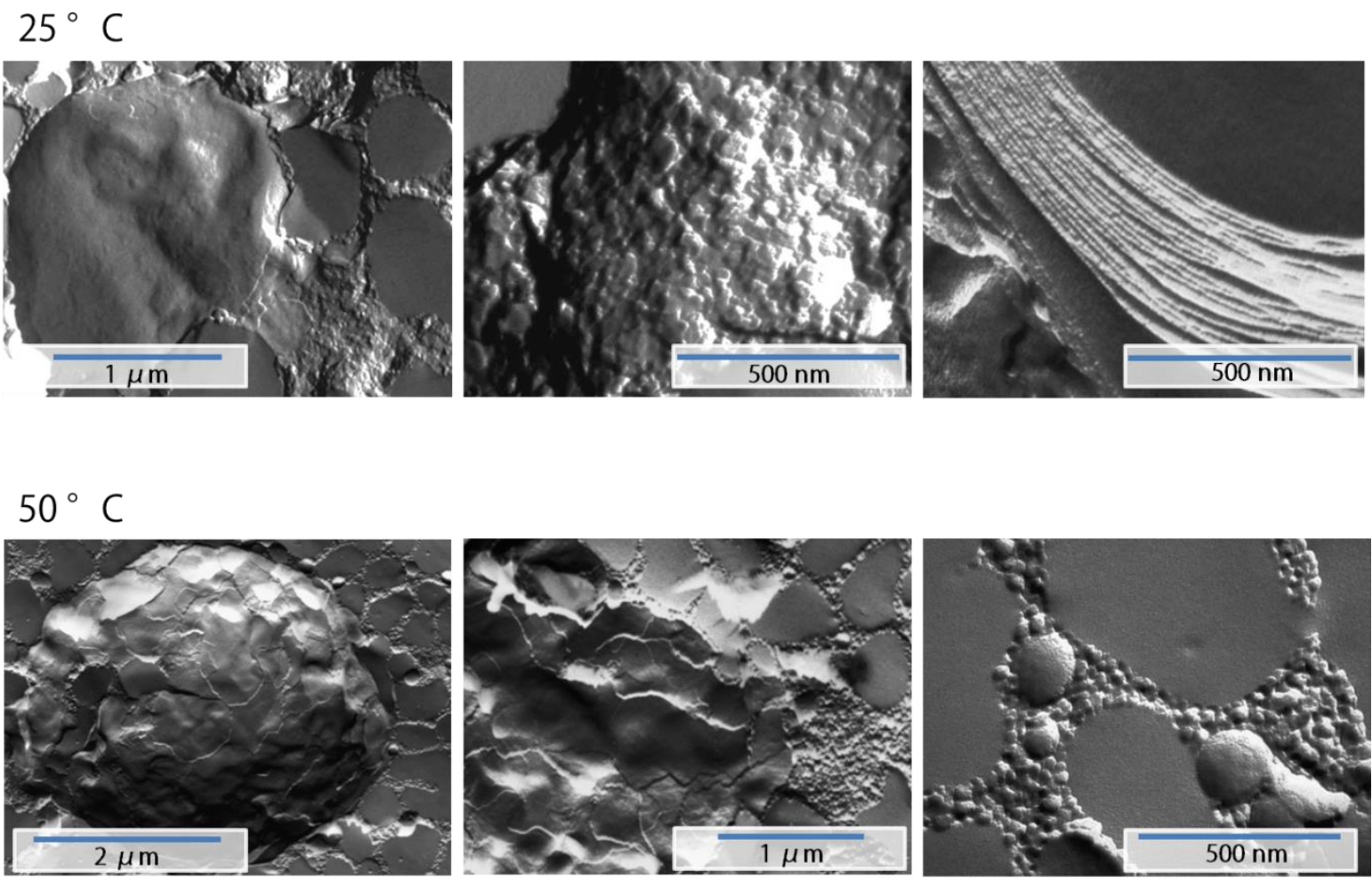

Figure 4. FFTEM pictures of PAMAM/DPPC system having $10^{-2}$ dendrimer/lipid molar ratio. 


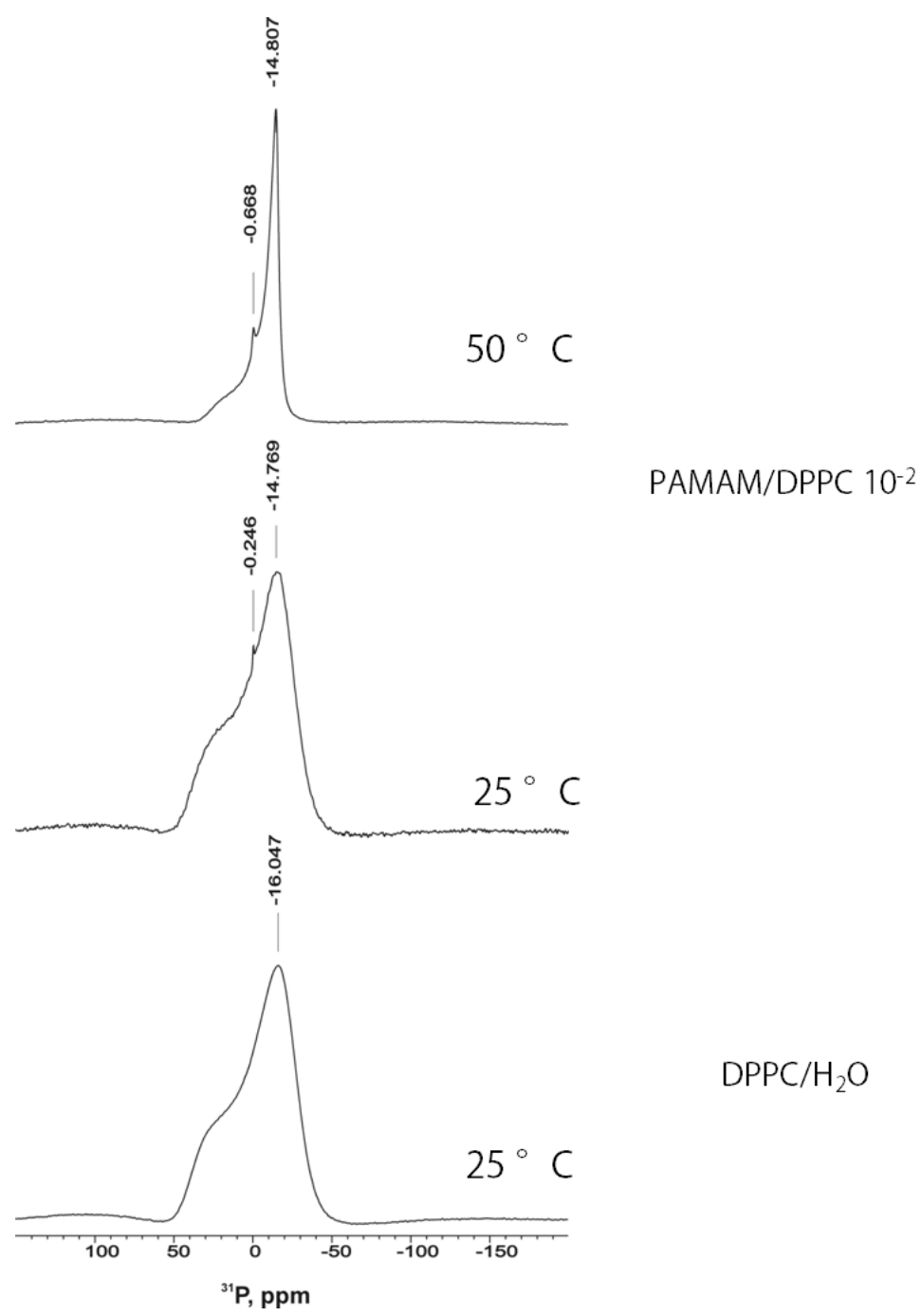

Figure 5. ${ }^{31} \mathrm{P}$ NMR spectra of fully hydrated DPPC MLVs and PAMAM-loaded DPPC-system having $10^{-2}$ dendrimer/lipid molar ratio.

\subsection{Changes on the molecular level}

In order to clarify the structural behavior of dendrimer-loaded DPPC systems on the molecular level, FTIR spectroscopic investigations were applied. ATR-FTIR spectra of pure DPPC and of PAMAM/DPPC systems with different concentrations are shown in Figure 6., compared to the spectrum of the neat PAMAM dendrimer recorded from aqueous solution. 


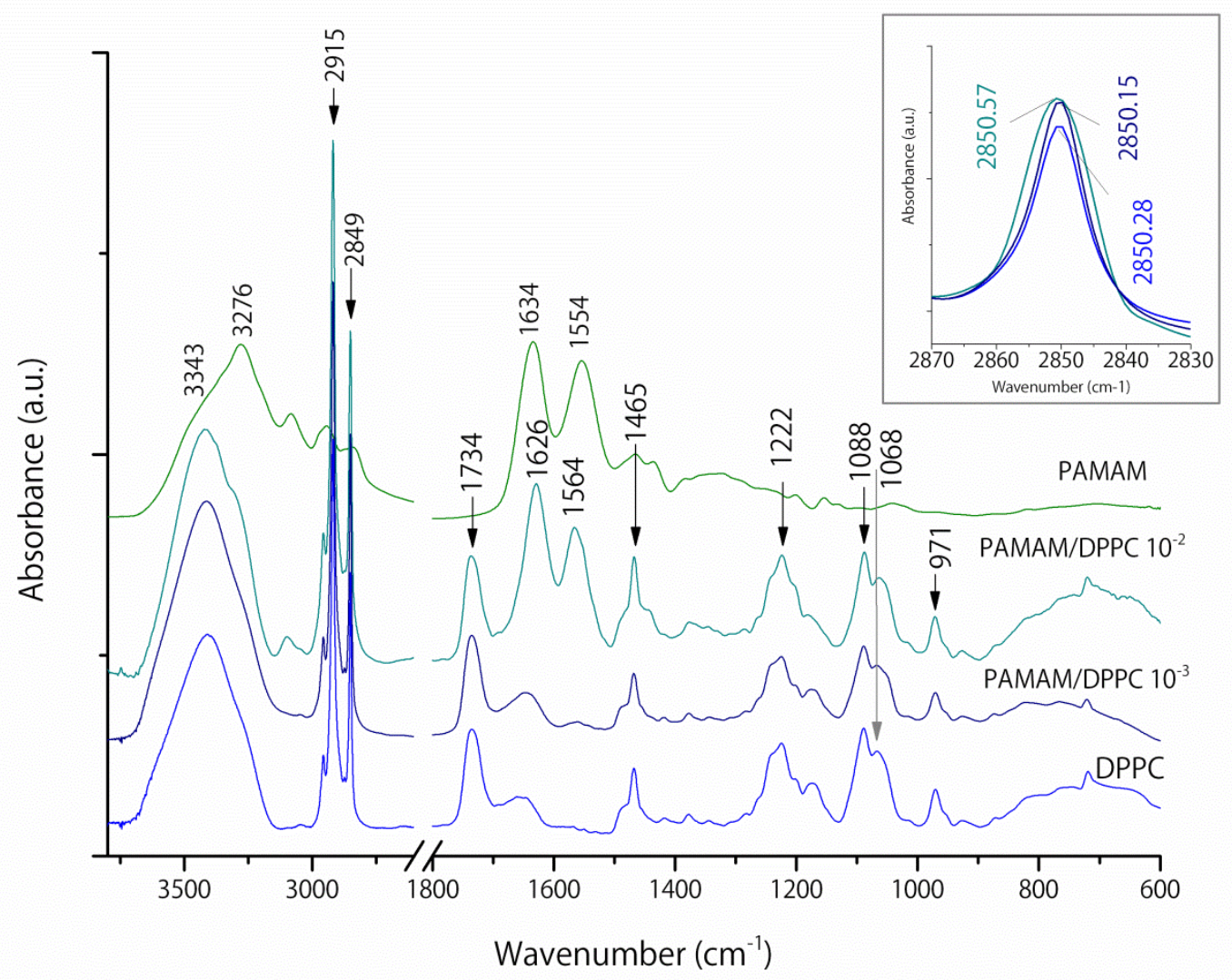

Figure 6. Infrared spectra of DPPC MLVs, PAMAM-loaded DPPC-systems and pure PAMAM dendrimer. All spectra are water subtracted. Arrow marked bands are characteristic for lipids. The symmetric $\mathrm{CH}_{2}$ stretching band wavenumber region (around $2850 \mathrm{~cm}^{-1}$ ) is enlarged in the inset.

The spectrum of the G5 PAMAM dendrimer (Fig. 6.) is dominated by two strong bands at 1634 and $1554 \mathrm{~cm}^{-1}$. The former can be assigned to the amide I mode of the polyamide units likely overlapped with the primary aliphatic amine deformation mode of the terminal $-\mathrm{NH}_{2}$ groups. The band at $1554 \mathrm{~cm}^{-1}$ belongs to the amide II mode. Considering the spectra of PAMAM/DPPC mixtures, a shift of the amide I and amide II bands can be observed as a result of the interaction between the dendrimer and the phospholipid bilayers. The downshift of the amide I frequency and the increase in the amide II band position suggest an increased level of hydrogen bonding of the dendrimer molecule [56].

Regarding the phospholipid structure, we focused on vibrations associated with the apolar alkyl chain region, characterized by the symmetric $\mathrm{CH}_{2}$ stretching vibration (2848-2853 $\left.\mathrm{cm}^{-1}\right)$; the polar-apolar interface or glycerol backbone region, featured by the carbonyl $\mathrm{C}=\mathrm{O}$ stretching mode $\left(1750-1720 \mathrm{~cm}^{-1}\right)$; and the polar headgroup region, described by the phosphate stretching vibrations $\left(1260-1000 \mathrm{~cm}^{-1}\right)$ [57].

The peak position of the symmetric methylene stretching $\left(\mathrm{v}_{\mathrm{s}} \mathrm{CH}_{2}\right)$ is a sensitive parameter to lipid order and packing, responsive to changes in the trans/gauche ratio of the lipid chains. There are no significant differences in the peak positions of the PAMAM loaded samples $\left(2850.15 \mathrm{~cm}^{-1}\right.$ and $2850.57 \mathrm{~cm}^{-1}$ Fig. 6. inset) compared to the pure DPPC MLVs $\left(2850.28 \mathrm{~cm}^{-1}\right)$ so only minor perturbations occur in the alkyl chain region. At the molar ratio of PAMAM/DPPC $10^{-2}$, however, a significant increase in the bandwidth was observed. 


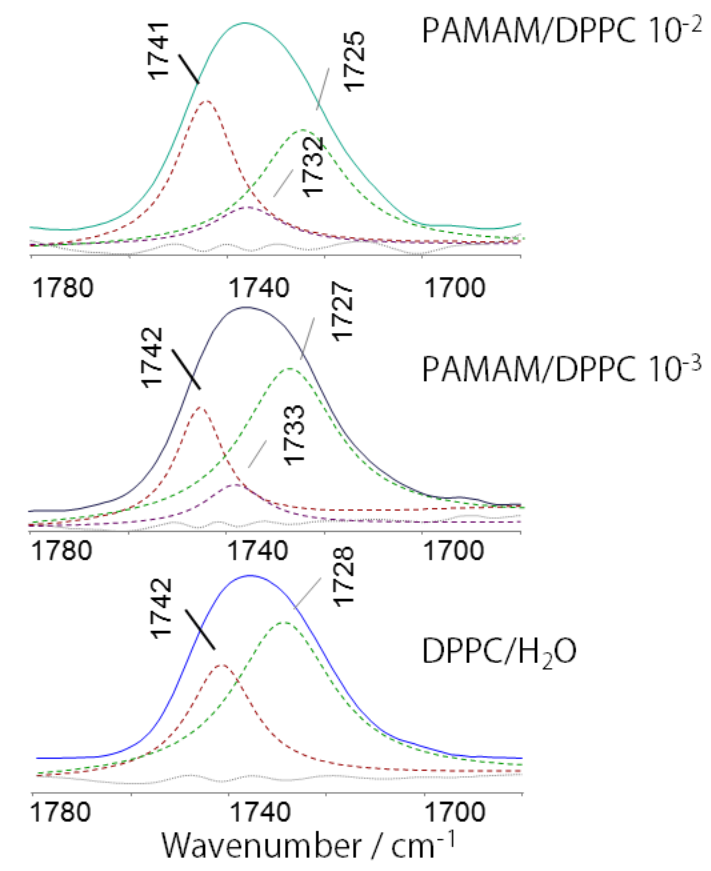

Figure 7. Infrared spectra of $\mathrm{C}=\mathrm{O}$ stretching region of DPPC MLVs, PAMAM-loaded DPPC-systems. Bands noted with dashed line correspond to the band components obtained by curve fitting procedure; dotted lines represent the residuals of the fittings.

The ester $\mathrm{C}=\mathrm{O}$ stretching vibration at $1750-1720 \mathrm{~cm}^{-1}$ is also sensitive to the changes in lipid structures. This broad band can be resolved for two different bands in highly hydrated bilayers: a high wavenumber band at $\sim 1740 \mathrm{~cm}^{-1}$, and a low wavenumber one at $\sim 1725 \mathrm{~cm}^{-1}$. Formerly, these components were assigned solely to $s n-1$ and $s n-2$ ester bonds of the glycerol backbone. Now it is widely accepted that these bands are assigned to differently hydrated subpopulations of both carbonyls [58]. Figure 7. shows the analysis of the results after curve fitting for the pure DPPC and the two PAMAM-loaded samples. By addition of G5 PAMAM dendrimers, the relative intensity of these two band components is changing. When dendrimers are added to DPPC, beside the non-hydrogen bonded $\mathrm{C}=\mathrm{O}\left(1742 \mathrm{~cm}^{-1}\right)$ and hydrogen bonded $\mathrm{C}=\mathrm{O}\left(\sim 1727 \mathrm{~cm}^{-1}\right)$ components, a new band around $1732 \mathrm{~cm}^{-1}$ appears after the curve fitting procedure. This new band component might be due to domains of lipid hydrated in a different way as a result of interactions with the dendrimers. This splitting of $\mathrm{C}=\mathrm{O}$ envelope is also evidenced in dry film spectra (not shown). At higher dendrimer concentration (PAMAM/DPPC $10^{-2}$ ) the $\mathrm{C}=\mathrm{O}$ contour is slightly changed due to the increased relative intensity of the non-hydrogen bonded $\mathrm{C}=\mathrm{O}$ band, indicating changes in the hydration level and in the dipole moment of the polar-apolar lipid interface, too [59].

No significant difference between pure DPPC and dendrimer loaded systems can be observed upon examining the asymmetric $\mathrm{PO}_{2}^{-}$vibration $\left(1223 \mathrm{~cm}^{-1}\right)$, which can serve as an indicator for the hydration state of the lipid molecules. The R-O-P-O-R' diester band $\left(1068 \mathrm{~cm}^{-1}\right.$ in the case of pure DPPC) is considered to reflect changes in the conformation of phospholipid headgroups [60]. At high concentration of PAMAM dendrimer $\left(10^{-2}\right)$, a new broad band centered at $1063 \mathrm{~cm}^{-1}$ appears. The PAMAM has also some bands in this region; nonetheless, subtracting the spectrum of pure dendrimer does not affect the above observation. This additional band can be witnessed also in dry film spectrum of PAMAM/DPPC $\left(10^{-2}\right)$ mixture (not shown). These changes in the phosphate headgroup vibrations confirm an intermolecular interaction of the phosphate groups with the terminal amine groups of PAMAM dendrimer directly or through a water molecule.

Temperature dependencies of the $\mathrm{CH}_{2}$ symmetric stretching frequency in the PAMAM-loaded DPPC-system with $10^{-2}$ dendrimer/lipid molar ratio were also investigated (see Supplementary information). There is no drastic changes when comparing the loaded system and the pure DPPC one, however, the main transition temperature interval is broadened and prolonged towards higher temperatures, conform to DSC curves. A slight increase in 
the $v_{\mathrm{s}} \mathrm{CH}_{2}$ band position indicates an increased number of gauche conformers relative to the pure DPPC. More interesting is the anomalous decrease of $v_{\mathrm{s}} \mathrm{CH}_{2}$ intensity in the transition temperature interval, suggesting the strong 'attachment' of lipid molecules to the dendrimers.

Summarizing the results of the ATR-FTIR measurements we can conclude that there is a traceable molecular interaction between the headgroups of the phospholipids and the PAMAM dendrimers when dendrimers are added in $10^{-2}$ molar ratio (regarding the changes of the amide I and amide II bands of PAMAM and the R-O-P-O-R' diester band of DPPC). This interaction affects also the hydration level of the polar-apolar lipid interface indicated by the $\mathrm{C}=\mathrm{O}$ stretching vibrations. Besides, a conformational disordering is introduced in the alkyl chain region according to the broadening of the ${ }_{\mathrm{s}} \mathrm{CH}_{2}$ band. Similar spectral changes were observed by Cieślik-Boczula et al. studying isotropic phases with high curvature in the DPPC structure induced by 3-pentadecylphenol [61]. The presence of an isotropic phase, experienced by our FTIR study is in accordance with the ${ }^{31} \mathrm{P}-\mathrm{NMR}$ results and might be due to small vesicles, observed on the FFTEM pictures.

\section{Conclusion}

The effect of $5^{\text {th }}$ generation, ethylenediamine-core PAMAM dendrimers on DPPC bilayers was investigated in different concentrations. Dendrimers added to the lipids in $10^{-3}$ molar ratio cause minor perturbations in the multilamellar structure and thermal behavior of liposomes. According to the DSC measurements PAMAM dendrimers most likely interact with the headgroup region of the bilayers and reduce the cooperativity of the alkyl chains during the main transition. This interaction causes also a loss in multilamellar correlation as it was shown by SAXS measurement.

Increasing the ratio of the dendrimers results in a significantly changed structure. The thermotropic character of the sample with $10^{-2}$ dendrimer/lipid molar ratio is different from that of the pure DPPC MLVs, as the pretransition vanishes and the main transition becomes a complex transition between different mesophases. These changes indicate a strong interaction between dendrimers and lipids which is able to alter the structure of both the headgroup and the alkyl chain region. ATR-FTIR investigations proved that the amine groups of PAMAM dendrimers do interact with the phosphate group of DPPC directly or through a water molecule. This interaction results also in an increase of conformational disorder of the alkyl chains. Structural changes were shown by SWAXS, FFTEM and ${ }^{31}$ P-NMR methods. The results reveal the coexistence of a highly swollen lamellar phase and a small proportion of dendrimers wrapped in lipid bilayers with high curvature. Taking in account all these results we propose that dendrimers attach to the surface of lipid bilayers through electrostatic forces and H-bonding. The surrounding lipids try to accommodate around the dendrimers to maximize headgroup-dendrimer interactions and so the bilayer surface becomes rough. Similar model was published recently by Åkesson et al [28]. Because of the net positive charge of the dendrimer-attached bilayers, electrostatic repulsion occurs and so the coherence of the layers will be weak or there will be no correlation depending on the local amounts of dendrimers. In the liquid crystalline phase however (at higher temperature), dendrimers can flatten out on the bilayer surface and so the whole layer becomes smoother. It seems plausible that not individual dendrimers but aggregates of them are able to be covered by phospholipid bilayers.

Our results give a direct proof and physico-chemical explanation on the former supposals that PAMAM dendrimers disrupt lipid membranes through a strong interaction with the phospholipids. Further detailed studies can provide information about the directions of modifications that make these lipid-dendrimer associates capable for drug delivery applications.

\section{Acknowledgements}

The authors thank Teréz Kiss for the FFTEM investigations. This work was supported by Hungarian Scientific Research Fund (OTKA, Hungary) and the National Innovation Office (NIH, Hungary) under grant agreement CNK-81056. 
References

$1 \quad$ O.M. Koo, I. Rubinstein, and H. Onyuksel, Nanomedicine 1 (2005) 193.

$2 \quad$ U. Boas and P.M.H. Heegaard, Chem. Soc. Rev. 33 (2004) 43.

3 H. Yang and W.J. Kao, J. Biomater. Sci. Polymer Ed. 17 (2006) 3.

$4 \quad$ J.B. Wolinsky and M.W. Grinstaff, Adv. Drug. Deliver. Rev. 60 (2008) 1037.

$5 \quad$ R. Esfand and D. A. Tomalia, Drug Discovery Today 6 (2001) 427.

$6 \quad$ Y. Kim and S.C. Zimmermann, Curr Opin Chem Biol 2 (1998) 733.

$7 \quad$ S. Svenson and D. A. Tomaila, Adv. Drug. Deliver. Rev. 57 (2005) 2106.

8 J. C. Roberts, M. K. Bhalgat and R. T. Zera, J. Biomed. Mater. Res. 30 (1996) 53.

9 R. Jevprasesphant, J. Penny, R. Jalal, D. Attwood, N. B. McKeown and A., D’Emanuele, Int. J. Pharm. 252 (2003) 263.

10 D. Fischer, Y. Li, B. Ahlemeyer, J. Krieglstein and T. Kissel, Biomaterials 24 (2003) 1121.

11 C. S. Braun, J. A. Vetro, D. A. Tomalia, G. S. Koe, J. G. Koe and C. R. Middaugh, J. Pharm.Sci. 94 (2005) 423.

12 C. Peetla, A. Stine, and V. Labhasetwar, Mol. Pharm. 6 (2009) 1264.

13 S. Hong, A. U. Bielinska, A. Mecke, B. Keszler, J. L. Beals, X. Shi, L. Balogh, B. G. Orr, J. R. Baker and M. M. Banaszak Holl, Bioconjugate Chem. 15 (2004) 774.

14 A. Mecke, I. J. Majoros, A. K. Patri, J. R. Baker, Jr., M. M. Banaszak Holl and B. G. Orr, Langmuir 21 (2005) 10348.

15 A. Mecke, D.K. Lee, A. Ramamoorthy, B.G. Orr and M.M. Banaszak Holl, Langmuir 21 (2005) 8588.

16 B. Erickson, S. C. DiMaggio, D. G. Mullen, C. V. Kelly, P.R. Leroueil, S. A. Berry, J. R. Baker Jr., B. G. Orr and M. M. Banaszak Holl, Langmuir 24 (2008) 11003.

17 C. V. Kelly, M. G. Liroff, L. D. Triplett, P. R. Leroueil, D. G. Mullen, J. M. Wallace, S. Meshinchi, J. R. Baker, Jr., B. G. Orr and M. M. Banaszak Holl, ACS Nano 3 (2009) 1886.

B. Wang, L. Zhang, S. C. Bae and S. Granick, Proc. Nat. Acad. Sci. U.S.A. 105 (2008) 18171.

S. Hong, R. Rattan, I. J. Majoros, D. G. Mullen, J. L. Peters, X. Shi, A. U. Bielinska, L. Blanco, B. G. Orr, J. R. Baker Jr. and M. M. Banaszak Holl, Bioconjugate Chem. 20 (2009) 1503.

R. Jevprasesphant, J. Penny, D. Attwood and A. D'Emanuele, J. Contorl. Rel. 97 (2004) 259.

H. Lee and R. G. Larson, J. Phys. Chem. B 110 (2006) 18204.

C. V. Kelly, P. R. Leroueil, E. K. Nett, J. M. Wereszcynski, J. R. Baker, Jr., B. G. Orr, M. M. Banaszak Holl and I. Andricioaei, J. Phys. Chem. B 112 (2008) 9337.

C. V. Kelly, P. R. Leroueil, B. G. Orr, M. M. Banaszak Holl and I. Andricioaei, J. Phys. Chem. B 112 (2008) 9346.

Li-Tang Yang and Xiaobo Yu, ACS Nano 3 (2009) 2171.

N. Karoonuthaisiri, K. Titiyevskiy and J.L. Thomas, Colloids and Surfaces B: Biointerfaces 27 (2003) $365-375.26$

A. Åkesson, K.M. Bendtsen, M.A. Beherens, J.S. Pedersen, V. Alfredsson and M.C. Gomez, Phys. Chem. Chem. Phys. 12 (2010) 12267-12272.

A. Åkesson, C.V. Lundgaard, N. Ehrlich, T.G. Pomorski, D. Stamou and M. Cardenas, Soft Matter 8 (2012) 8972-8980.

A. Åkesson, T.K. Lind, R. Baker, A. Hughes and M. Cardenas, Langmuir 28 (2012) 13025-13033.

F. Ruggeri, A. Åkesson, P.-I. Chapuis, C.A. Skrzynski Nielsen, M.P. Monopoli, K.A. Dawson, T.G. Pomorski and M. Cardenas, Soft Matter 9 (2013) 8862-8870. GUV+G6+BSA FM

Gregory Gregoriadis ed., Liposome Technology Third Edition Vol I, Informa Healthcare USA, Inc. 2007 
K. Gardikis, S. Hatziantoniou, K. Viras, M. Wagner and C. Demetzos, Int. J. Pharm. 318 (2006) 118.

D. Wrobel, M. Ionov, K. Gardikis, C. Demetzos, J.P. Majoral, B. Palecz, B. Klajnert and M. Bryszewska, Biochim. Biophys. Acta 1811 (2011) 221.

P.E.S. Smith, J.R. Brender, U.H.N. Dürr, J. Xu, D.G. Mullen, M.M. Banaszak Holl and A. Ramamoorthy, J.Am.Chem.Soc. 132 (2010) 8087.

B. de Kruijff, A.J. Verkley, C.J.A. van Echteld, W.J. Gerritsen, C. Mombers, P.C. Noordam and J. de Gier, Biochim. Biophys. Acta 555 (1979) 200.

G. Goerigk, Electronics and Computer Upgrades at ASAXS Beamline Jusifa. HASYLAB, Hamburg, Germany, 2006.

U. Vainio, T. Schubert, S. Botta, J. Blume, A. Wacha, M. Lohmann, Th. Kracht and R. Gehrke, New in situ SAXS/WAXS setup and other refurbishments at the ASAXS beamline B1. HASYLAB, Hamburg, Germany, 2006.

H.-G. Haubold, K. Gruenhagen, M. Wagener, H. Junbluth, H. Heer, A. Pfeil, H. Rongen, G. Brandenberg, R. Moeller, J. Matzerath, P. Hiller and H. Halling, Rev. Sci. Instrum. 60 (1989) 1943.

A. Bergmaschi, A. Cervellino, R. Dinapoli, F. Gozzo, B. Henrich, I. Johnson, P. Kraft, A. Mozzanica, B. Schmitt and X. Shi, J. Synchrotron Rad. 17 (2010) 653.

Ch. Broennimann, E. F. Eikenberry, B. Henrich, R. Horisberger, G. Huelsen, E. Pohl, B. Schmitt, C. Schulze-Briese, M. Suzuki, T. Tomizaki, H. Toyokawa and A. Wagner, J. Synchrotron Rad. 13 (2006) 120.

B. Henrich, A. Bergamaschi, C. Broennimann, R. Dinapoli, E.F. Eikenberry, I. Johnson, M. Kobas, P. Kraft, A. Mozzanica and B. Schmitt, Nuclear Istruments and Methods in Physics Research A 607 (2009) 247.

C. Grabielle-Madelmont and R. Perron, J. Colloid Interf. Sci. 95 (1983) 471.

M. Pantusa, R. Bartucci and L. Sportelli, Colloid Polym. Sci. 285 (2007) 649.

B.G. Auner, M.A.A. O’Neill, C. Valenta and J. Hadgraft, Int, J. Pharm 294 (2005) 149.

P.L. Yeagle ed., The Structure of Biological Membranes - Second Edition; CRC Press 2004

B.G. Tenchov, H. Yao and I. Hatta, Biophys. J. 56 (1989) 757.

A. Bóta, Z. Varga and G. Goerigk, J. Appl. Cryst. 40 (2007) 259.

R. Zhang, R. M. Suter and J.F. Nagle, Phys. Rev. E 50 (1994) 5047.

A. Guinier.: X-ray diffraction. San Francisco: Freeman, 1963.

G. Pabst, R., Koschuch, B. Pozo-Navas, M. Rappolt, K. Lohner and P. Laggner, J. Appl. Cryst. 36 (2003)1378.

N.S. Myshakina, Z. Ahmed and S.A. Asher, J Phys Chem B 112 (2008) 11873.

R.N.A.H. Lewis and R.N. McElhaney, Vibrational Spectroscopy of Lipids, in Handbook of Vibrational Spectroscopy (eds: JM Calmers and P Griffiths), John Wiley \&Sons, Inc. 2001 pp. 3784-3801. 1828 (2013) 661.

61 K. Cieślik-Boczula and A. Koll, Biophys. Chem. 140 (2009) 51-56. 